\title{
Intelligent Offer Model for Game Online Item with Principle Hybrid Filtering Together with Categorical Data Technique
}

\author{
Khammapun Khantanapoka \\ Department of Multimedia Technology, Faculty of Science, \\ Chandrakasem Rajabhat University, Thailand. \\ doramon86@hotmail.com
}

\begin{abstract}
This paper focus to Intelligent offer pattern, The commonly used raw material, wearing: costumes, earrings, gloves, gloves, boots, shoes, costumes, hair, necklace, two hands, costume masks, hats, costumes, weapons, clothes, watches, rings, plaques and feature character. Robot and various tools are built to response of players. One of the expectations of most players is items selection which is selected from the User Interface. It is obtrusive noise of view screen while players select a few of the items. Object Items of games offer to each player overlap on the screen. It helps reduce event to switch the screen to maximize revenue from the purchase, sale, and exchange object items in the online game. we use Double Two dimension Principle Component Analysis for the feature extraction data behavior which is behavior a repeated in each player such as character of clothes, characteristic of choose activity , characteristic of use game items. We built Collaborative group pattern (CGP) and analysis player separate to each corporative group. Adding create offer game items in each corporative group. Each player will receive the best offers. The game operator has the opportunity to sell game items online as possible by without interrupting game play. The results of experiment showed that this method can offer a game item which is player want to sequence in the top of the list, and average 11.41 percentages is garbage items which player does not want, by game items which each player need to be in the order of 1-10 of menu.
\end{abstract}

Keyword-collaborative group, Collaborative Filtering.

\section{INTRODUCTION}

Games Online is a video game played on a computer network on the Internet. Online games are similar to multi-player games over the Internet. (Multiplayer) games online most of the game, MMO (Massive Multiplayer Online), or is the game for many players to get a number of players have been tremendous in the area, one (from 100 persons or more) games have been very popular since. Social players are fun to play with friends than playing alone. Online multiplayer game with beautiful graphics. So it is appealing to people to play games online. Games and activities. Are added to the map in the game, including a monster new weapons and other continuously.
However, Game Online can generate revenue from the sale of game item.

Game Item (GI) is an object within the game world that can be collected by a player or, occasionally, a non-player character. These items are sometimes called pick-ups. The most of game items are often beneficial to the player character, but some games contain detrimental items, such as cursed pieces of armor that confers a negative bonus to the wearer, and cannot be removed until the curse itself is lifted; the means to do this may be costly or require a special item. Some items may also be of absolutely no value to the player. Items are especially prevalent in role-playing games, as they are usually necessary for the completion of quests or to advance through the story.

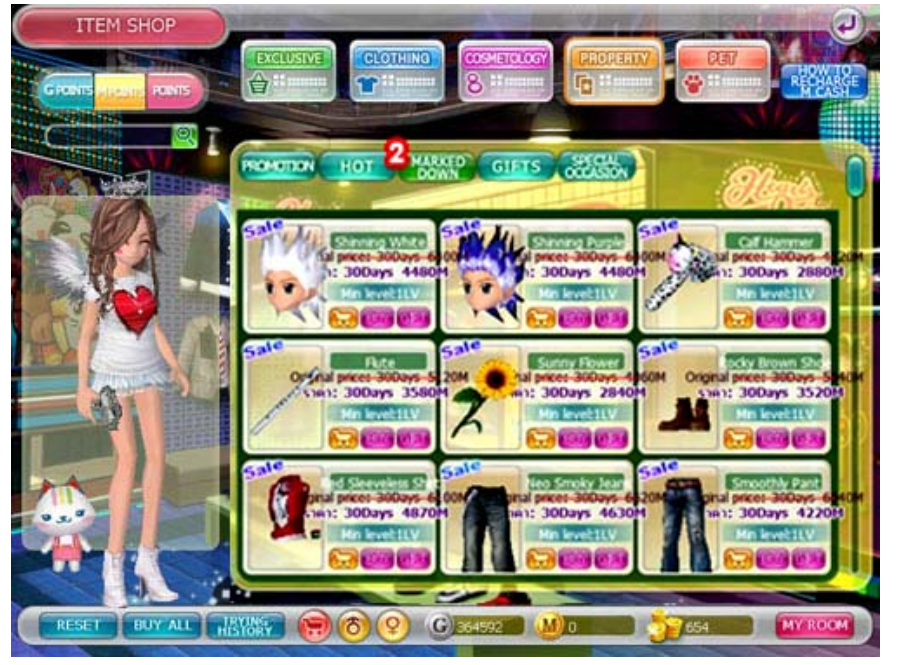

Figure 1. Example of game items which sever operation offer to player in each scene of game which is effect to play slower and visible disturbances players.

Treasure such as coins, rings, gems or jewelry is another common item. These are often used to determine the player's score. In some games, particularly those with an over world map, players can take these items to a shop-like place and exchange them for new abilities or equipments. Usually, such treasure items are found in small quantities as one progress through a level. However, by exploring, players can often find secret areas containing a large number of them. In some platforms, particularly those with a hit counter. The game 
offer to favorite items for each player. Online game is a game which played on multiplayer or MMO. It is the game for many players ผ่าน reference a ID of players. The various players will density in the same area of virtual reality. Online games have been very popular with reason interactive between social players are fun to play than playing alone, games and activities, maps, monster, new weapons and continuously of story. These object items are disturbing sight but it built a fun and individual character to the player. It assists decision to buy from distributors game items. The development of artificial intelligence became to tools for offered game items such as match the likes of players, color, style, pets and game items associated with the style of each player. The players are avoid pop-up deep to find game items on online games. The offer system of the intelligent game items will presented to each player which is less than to show all game items. The recommendation system of game items will start simultaneously with electronic commerce. In general,

\section{LITERATURE REVIEW}

The guidance system can be divided three ways. (1) Use Collaborative Filtering (2).Use filter the data by looking at the content. (Content-based Filtering) and (3).Use Hybrid filtering technique. In this research, we use collaborative filtering method in activity offer game items such as buying clothes and other game items. From assumed, a group of players in the same group should a favorites in same game items. The players will be the offer follow frequency from the some information of each player that the game items is different characteristics such as color, shape and structure of game items. The game Items selected by the frequency of each pattern which is keep as a rating (Rating) to the Item. The basis of diversity wills clustering by statistic relation some information compare with other players. The problem is important in everyday due to user access same pattern. We use statistic model for offer the game Items for each scenes which is difference by information similarity of each player. The large amount of the game Items will allow the system to offer cluster of game items which is high quality. We focus to find people who have the similar preferences on players. We are a group of players with the same characteristics with online analysis. However, we do not know the other players before. Grouping of players help online game operator decided to offer some game items on screens which meets the needs of the players. It helps to support the purchase of game items while reducing visual distractions and reduce complexity of menu command.

\section{EXPERIMENTAL}

Each player will be the accumulated frequency of selection each game items which has unique different such as color, and structure to build a relationship identify with the identity information of the player. We use this information built pattern recognition of machine from feature extraction process. The frequency of selected in each player will stored as a rating for choose offer game items of machine. The machine will choose some offer items base on diversity inside prefer of individual players. But filtering methods rely on the use of sharing information has mistake in many respects such as sparsely problem, missing value. The most issue is each player chooses to use game items for some scenes. There is the large information empty which is effect to offer system low quality. The incorrect of computation are effect to the loss of customers. The high error will negative to player losing the opportunity to buy game items and not enjoy for game. The input of this system is frequency of selected for each game item by each player in each Level. We consider data of choose game items to prefer in individual. We analyze behavior selection and create a Collaborative group pattern (CGP) using $2 \mathrm{D}^{2} \mathrm{PCA}$ algorithm. Therefore, this research proposes methods to solve the problem of sparse data using statistical methods. Our methods applied to the collaborative filter techniques together with missing value to predict satisfaction with game items. This method uses statistical methods to find the information the user is a member of Collaborative group pattern which liking game items similar to the offer appropriate. The player data that is nearby will be analyzed and offer game items directly to the needs of the sale goal.

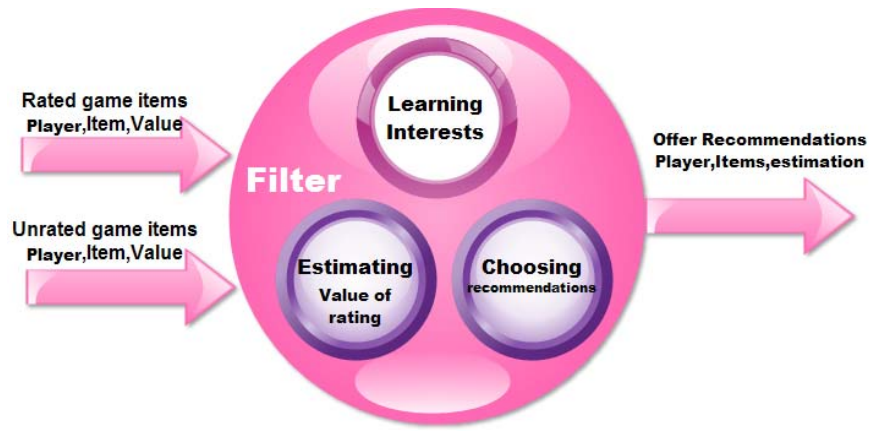

Figure 2. model of Collaborative Filtering technique for separate player to group representation.

\section{A. Collaborative Filtering:}

Combine Content-based and Collaborative Filtering type A

Collaborative filtering (CF) is a technique used by some recommender systems. Collaborative filtering has two senses, a narrow one and a more general one. In general, collaborative filtering is the process of filtering for information or patterns using techniques involving collaboration among multiple agents, viewpoints, data sources, etc. Applications of collaborative filtering typically involve very large data sets. Collaborative filtering methods have been applied to many different kinds of data including: sensing and monitoring data, such as in mineral exploration, environmental sensing over large areas or multiple sensors; financial data, such as financial service institutions that integrate many financial sources; or in electronic commerce and web applications where the focus is on user data, etc. The remainder of this discussion focuses on collaborative filtering for user data, although some of the methods and approaches may apply to the other major applications as well. We use Similarity measure: Pearson Correlation Coefficient which represents to equation as follow:Collaborative Filtering type A 


$$
k_{c i}=\frac{\sum_{j \in i_{c i}}\left(r_{c j}-\bar{r}_{c}\right)\left(r_{i j}-\bar{r}_{j}\right)}{\sqrt{\sum_{j \in I_{c i}}\left(r_{c j}-\bar{r}_{c}\right)^{2} \sqrt{\sum_{j \in I_{c i}}\left(r_{i j}-\bar{r}_{j}\right)^{2}}}}
$$

Recommendations Computation: weighted sum of ratings

$$
\hat{r}_{c j}=\bar{r}_{C}+\frac{\sum_{j \in U_{c j}}\left(r_{i j}-\bar{r}_{i}\right) k_{c i}}{\sum_{i \in U_{c j}}\left|k_{c i}\right|}
$$

The computing will estimates for missing ratings by Contentbased Filtering method for each player. It searching for likeminded players computing coefficient $\mathrm{k}_{\mathrm{ci}}$ between current and $\mathrm{i}$-th user (only from ratings) computing coefficient $\mathrm{k}_{\mathrm{ci}}$ between current and i-th user (from both ratings and estimates) For new recommendations computation will using ratings (with coefficients $\mathrm{k}_{\mathrm{c}}$ ) and also ratings with estimates (with coefficient $\mathrm{k}_{\mathrm{ci}}{ }^{\prime}$ ) as weights in weighted sum of ratings and estimates.

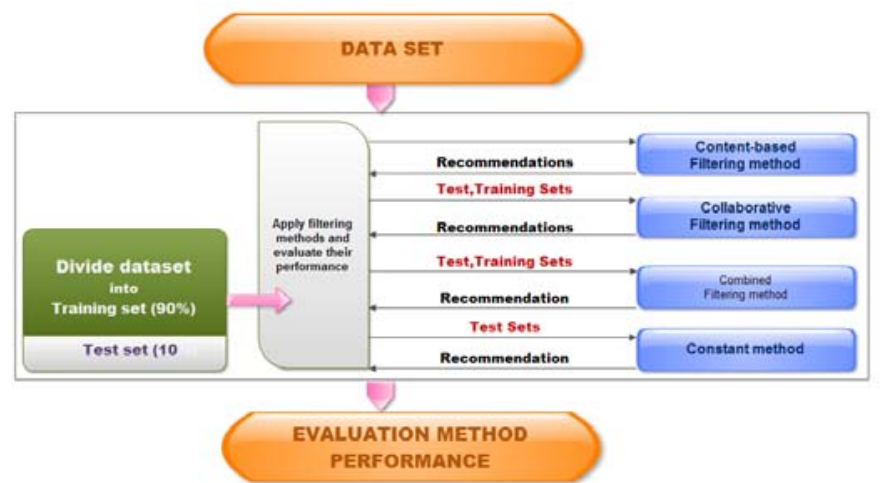

Figure 3. Show Experiment with Combination of Content-based and Collaborative Filtering

We use building new Collaborative group pattern (CGP) with Double Two Dimension in Principle Component Analysis Algorithm which is analysis data of interactive activity using operation follow as ;

1.Metrics= Coverage percentage of items for which the method is able to compute estimates

$$
\text { 2.Accuracy }=\frac{|R \cap L|+|\bar{R} \cap \bar{L}|}{|L|+|\bar{L}|}
$$

while $\mathrm{R}$ as set of recommended and $\mathrm{L}$ as set of liked items

$$
\begin{aligned}
& \text { 3.F-Measure }= \frac{2 \text { Precision.Recall }}{\text { Precision.+Recall }} \\
& \text { While Precision }=\frac{|R \cap L|}{|R|} \text { and Recall }=\frac{|R \cap L|}{|L|} \\
& \text { 4.NMAE }=\frac{\sum \mid r_{i j}-\hat{r_{i j} \mid}}{n . s}
\end{aligned}
$$

Combine Content-based and Collaborative Filtering type B.

Similarity measure: Pearson Correlation Coefficient which represents to equation as follow: Collaborative Filtering type
B using Recommendations Computation: weighted sum of ratings and estimates as equation:

$$
\hat{r}_{c j}=\bar{r}_{C}+\frac{\sum_{j \in U_{c j}}\left(r_{i j}-\bar{r}_{i}\right) k_{c i}+\sum_{i \in U_{c j}^{\prime}}\left(r_{i j}^{C B F}-\bar{r}_{i}\right) k_{c i}^{*}}{\sum_{i \in U_{c j}}\left|k_{c i}\right|+\sum_{i \in U_{c j}^{\prime}}\left|k_{c i}^{*}\right|}
$$

Recommendations computation: weighted sum of ratings

- $\quad$ Content-based Filtering Method (CBF) vector representation - weighted keywords (TF-IDF), estimation computation: normalized dot product of document and profile vectors

- Collaborative Filtering (CF) Pearson correlation coefficient weighted sum of ratings

- Combination of CF and CBF Pearson correlation coefficients

\begin{tabular}{|c|c|c|c|c|c|}
\hline Scene & (item 1) & (item 2) & (item 3) & (item 4) & (item 5) \\
\hline Player 1 & empty & 5 & 4 & 5 & 2 \\
\hline Player 2 & 4 & (a) & 3 & 5 (b) & 2 \\
\hline Player 3 & 4 & Empty (a) & empty & 3 (b) & 2 \\
\hline Player 4 & 4 & 5 & 3 & 3 & 4 \\
\hline Player 5 & empty & 3 & 4 & 3 & 2 \\
\hline
\end{tabular}
weighted sum of ratings and CBF estimations

- Constant Method

Player profile :

The choice of one user ID convert to the player profile of the player ID for generate to Collaborative group pattern (CGP).

(a) Not Collaborative items and (b) Collaborative items

B. Finding a player in the group pattern.

This step is the process of finding a player in the group with the selection game Items and preferences similar to those used to select the same game Items Level. If you find that one of the ID Player 2 with a passion similar to ID Player 3.Or not, we have to consider the scores and the frequency of selection of the ID Player 2 and ID Player 3 is the same which is called game items related.

\section{Built new Collaborative group pattern (CGP)}

We built this information to new Collaborative group pattern (CGP) using (2D) ${ }^{2}$ PCA algorithm.

\section{Double Two Dimension in Principle Component Analysis Algorithm (2D) ${ }^{2} P C A$}

$2 D^{2}$ PCA learns an optimal matrix $X$ from a set of training data reflecting information between rows of data, and then projects an $m$ by $n$ data $A$ onto $X$, yielding an $m$ by $d$ matrix $Y=A X$ Similarly, the alternative 2DPCA learns optimal matrix $Z$ reflecting information between columns of data, and then projects $A$ onto $\mathrm{Z}$, yielding a $q$ by $n$ matrix $B=Z^{T} A$. In the following, we will present a way to simultaneously use the projection matrices $X$ and $Z$ Suppose we have obtained the 
projection matrices $X$ and $Z$ projecting the $m$ by $n$ data $A$ onto $X$ and $\mathrm{Z}$ simultaneously, yielding a $q$ by $d$ matrix $C$.

$$
C=Z^{T} A X
$$

The matrix $C$ is also called the coefficient matrix in data representation, which can be used to reconstruct the original data $A$, by

$$
\hat{A}=Z C X^{T}
$$

When used for data recognition, the matrix $C$ is also called the feature matrix. After projecting each training data $A_{k}(k=1,2, \ldots M)$ onto $X$ and $Z$, we obtain the training feature matrices $C_{k}(k=1,2, \ldots M)$. Given a test data set the distance between $C$ and $C_{\mathrm{k}}$ is defined by

$$
d\left(C, C_{k}\right)=\left\|C-C_{k}\right\|=\sqrt{\sum_{i=1}^{q} \sum_{j=1}^{d}\left(C^{(i, j)}-C_{k}^{(i, j)}\right)^{2}}
$$

Equation 7. where the calculated value indicates that two players is similar. However, if the calculated value is less than two players have a few similarities. This system will build a list appropriate item out to the players. Targets are ranked as Top-N list Item by scores which is calculated for player in each Collaborative group pattern (CGP).

$$
\operatorname{Sim}\left(u_{i}, u_{j}\right)=\frac{\sum_{k=1}^{n}\left(r_{u i, k}-\bar{r}_{u i}\right) \times\left(r_{u j, k}-\bar{r}_{u j}\right)}{\sigma_{u i} x \sigma_{u j}}
$$

while $\operatorname{Sim}\left(u_{i}, u_{j}\right)$ as value of the similarity between player $u_{i}$ and $\mathrm{u}_{j}$.

$r_{u i, k}$ as value of the likelihood that player $u_{i}$ for a game item No.k.

$r_{u j, k}$ as value of the likelihood that player $u_{j}$ for a game item No.k.

$\bar{r}_{u i}$ as average preference of the player $u_{i}$ for whole game item which the players $u_{i}$ had been selected.

$\bar{r}_{u j}$ as average preference of the player $u_{j}$ for whole game item which the players $u_{j}$ had been selected.

$\sigma_{u i}$ as variance of value of the likelihood that player $u_{i}$ for whole game item which the players $u_{i}$ had been selected.

$\sigma_{u j}$ as variance of value of the likelihood that player $u_{j}$ for whole game item which the players $u_{j}$ had been selected.

$n$ as collaborative items

\section{E. The predicted process for offer game items in each CGP}

By considering the similarity selection between one player to another player game Items by ID is the set of members with similar preferences similar become same group of Collaborative group pattern (CGP)

$$
p\left(u_{i, k}\right)=\bar{r}_{u i}+\frac{\sum_{j=1}^{m} w_{u i, u j}\left(r_{u j, k}-\bar{r}_{u j}\right)}{\sum_{j=1}^{m} w_{u i, u j}}
$$

while

$p\left(u_{i, k}\right)$ as the value of prediction for the game Items No.k of player $u_{i}$

$\bar{r}_{u j} \quad$ as average game items 's score of the player $u_{j}$

$m$ as member of Best-Neighbor

$r_{u j, k}$ as game items 's score of player $u_{j}$ give to game items

No.k

$w_{u i, u j}$ as value of the similarities between players $u_{i}$ and $u_{j}$

Similar angular measurement system is expected to provide more accurate method to measure the similarity of the Euclidean section.

$$
M A E=\frac{\sum_{k=1}^{n}\left|p_{k}-q_{k}\right|}{n}
$$

while

$p_{k}$ as score of game items No.k of players in data set.

$q_{k}$ as score of game items No.k of players which return from predicted process of game.

F. Missing value in empty data due to some offer player not being selected from items list.

\begin{tabular}{|c|c|c|c|c|c|}
\hline & (item 1) & (item 2) & (item 3) & (item 4) & (item 5) \\
\hline Average & 5 & 5 & 3.5 & 4.01 & 6.47 \\
\hline Variance & 0 & 1 & 0.25 & 0.86 & 0.86 \\
\hline & $\operatorname{inv} \operatorname{sim}\left(u_{i} u_{j}\right)=\frac{1}{1+\sqrt{\sum_{k=1}^{n}\left(r_{u i, k}-r_{u j, k}\right)^{2}}}$
\end{tabular}

Example of filling the missing value from calculate with equation (10)

\begin{tabular}{|c|c|c|c|c|c|}
\hline Scene & (item 1) & (item 2) & (item 3) & (item 4) & (item 5) \\
\hline Player 1 & $4^{*}$ & 5 & 4 & 5 & 2 \\
\hline Player 2 & 4 & 3 & 3 & 5 & 2 \\
\hline Player 3 & 4 & $3^{*}$ & $3^{*}$ & 3 & 2 \\
\hline Player 4 & 4 & 5 & 3 & 3 & 4 \\
\hline Player 5 & $4^{*}$ & 3 & 4 & 3 & 2 \\
\hline Player $n$ & $\ldots$ & $\ldots$ & $\ldots$ & $\ldots$ & $\ldots$ \\
\hline
\end{tabular}

\section{IV.RESULT OF EXPERIMENTAL}

We develop online games, java game programming by MMOG engine. We test with 218 players from 120 game Items base on 35 scenes. The results of the experiment showed; the players can separation 21 groups which each group will offer game items for selection in time only 32 species. We explore the satisfaction of players in each time which AI presentation about offer game items. All players have a very good level of satisfaction. Since players can play the game faster. And game items that each player need to be in the order of 1-10 of menu. In the scenes level 1-9, the game items that need to be fragmented, by average 54.06 percent of game items is garbage items which player does not want. In the scenes level 10-18, game items that player want has sequence in the middle of the list and average 31.75 percent is garbage items which player does not want. In the scenes level 19-30, game items that player want has sequence in the top of 
the list and average 18.73 percent is garbage items which player does not want. In the scenes level 30-35, game items that player want has sequence in the top of the list and average 11.41 percent is garbage items which player does not want and game items that each player need to be in the order of 110 of menu.

\section{CONCLUSION}

We can apply collaborative filtering to the application in other games or use the Games to promote the business-online. So that business can be operate in conjunction with the game.

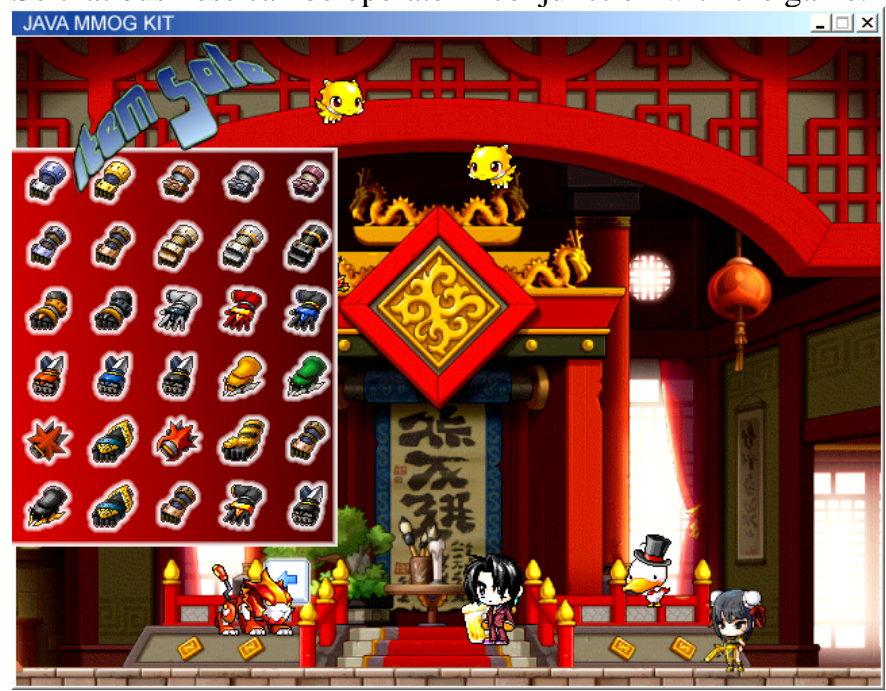

Figure 3. game online with Java programming for Gloves items which develop for test in our research. We use tools bennedstory web.

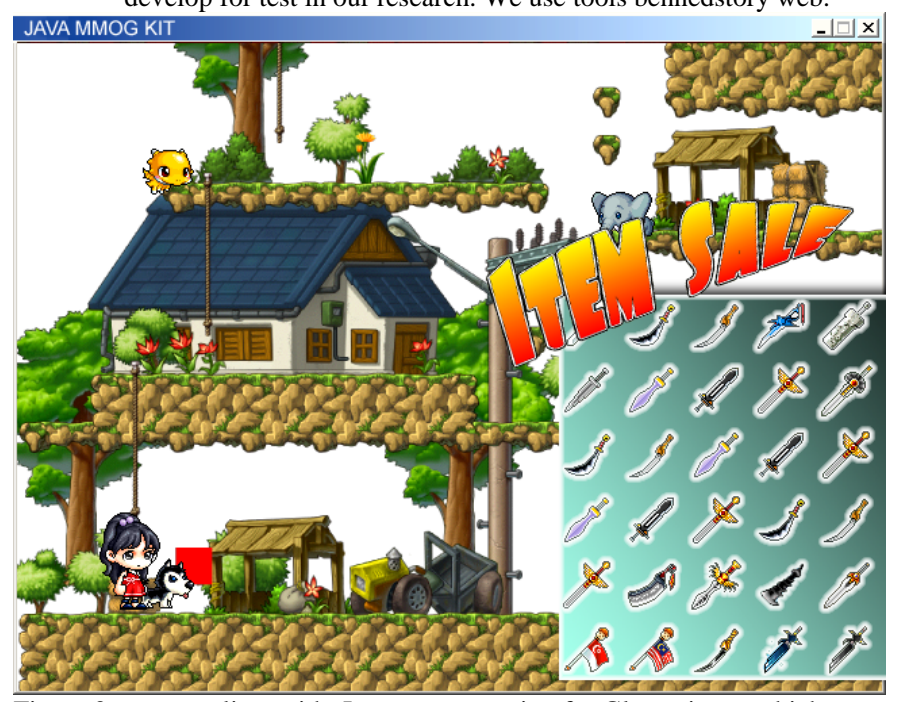

Figure 3. game online with Java programming for Gloves items which develop for test in our research. We use tools bennedstory web

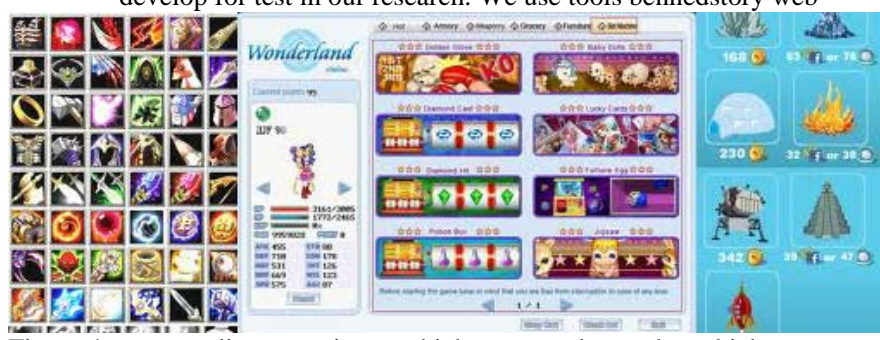

Figure 4. game online game items which are prevalent today which

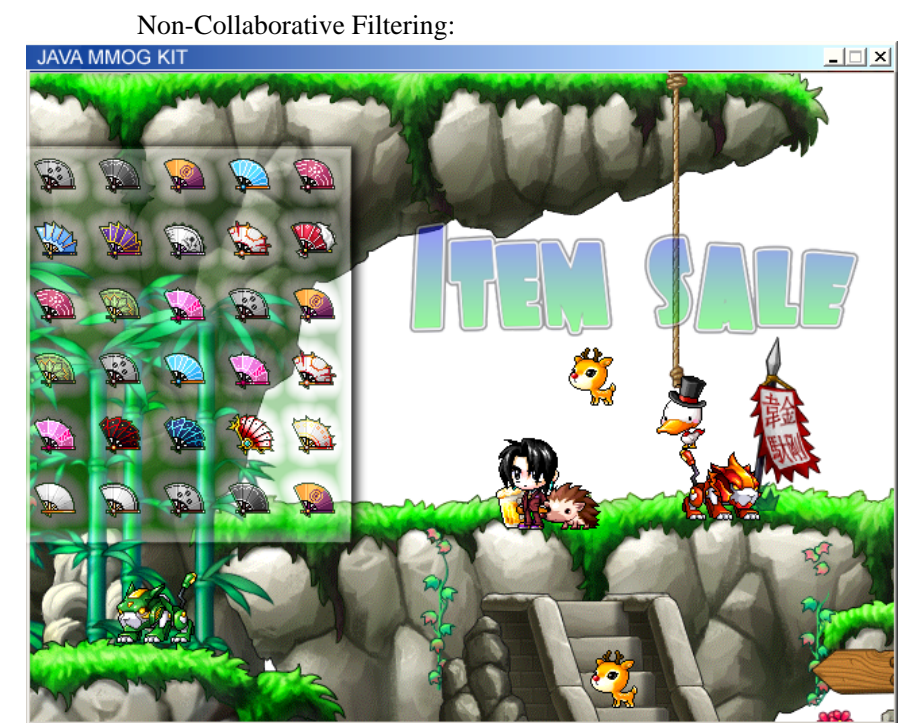

Figure 5. game online with Java programming for Gloves items which develop for test in our research. We use tools bennedstory web : http://www.maplesimulator.com/

PREFERENCE

[1] Luiz Andre Barroso, Jerey Dean, and Urs Holzle. Web search for a planet: The Google Cluster Architecture. IEEE Micro, 23(2):22\{28, March/April 2003.

[2] Wray Buntine and Sami Perttu. Is Multinomial PCA Multi-faceted Clustering or Dimensionality Reduction? In 9th International Workshop on Articial Intelligence and Statistics (AISTAT-2003), 2003.

[3] M. E. Brand. Incremental singular value decomposition of uncertain data with missing values. In Proceedings of the European Conference on Computer Vision (ECCV), volume 2350, pages 707\{720, May 2002.

[4] John S. Breese, David Heckerman, and Carl Kadie. Empirical Analysis of Predictive Algorithms for Collaborative Filtering. In Proceedings of the Fourteenth Annual Conference on Uncertainty in Articial Intelligence, pages 43\{52, July 1998.

[5] W. Buntine. Variational extensions to EM and multinomial PCA. In Proceedings of the European Conference on Machine Learning ECML, 2002.

[8] Hendrik Blockeel, Luc De Raedt, and Jan Ramon. Top-down induction of Clustering trees. In J. Shavlik, editor, Proceedings of the $15^{\text {th }}$ International Conference on Machine Learning, pages 55\{63. Morgan Kaufmann, 1998

[9] M. E. Brand. Incremental singular value decomposition of uncertain data With missing values. In Proceedings of the European Conference on Computer Vision (ECCV), volume 2350, pages 707\{720, May 2002.

[6] D. Blei, A. Ng, and M. Jordan. Latent Dirichlet Allocation. In Proceedings of the 14th Conference on Advances in Neural Information Processing Systems (NIPS 14), 2002.

[7] D. Blei, A. Ng, and M. Jordan. Latent Dirichlet allocation. Journal of Machine Learning Research, 3:9931022, January 2003.

[8] K. Goldberg, T. Roeder, D. Gupta and C. Perkins, "Eigentaste: A Constant Time Collaborative Filtering Algorithm”, InformationRetrieval 4 (2001), 133-151.

[9] J. L. Herlocker, J. A. Konstan, A. Borchers and John Riedl, “An Algorithmic Framework for Performing CollaborativeFiltering”,Proc. 22ndACM SIGIRConference on Information Retrieval, pp. 230-237, 1999.

[10] J. Konstan, B. Miller, D. Maltz, J. Herlocker, L. Gordon and J. Riedl, "GroupLens:ApplyingCollaborativeFilteringtoUsenetNews", Communicationsof the ACM 40 (1997), 77-87,www.grouplens.org.

[11] C.L. Lawson andB.J. Hanson, Solving Least Squares Problems, PrenticeHall, 1974

[12] G. Linden, B. Smith and J. York, “Amazon.com Recommendations: Item-to-itemCollaborative Filtering”, IEEE InternetComputing 7 (2003), 76-80.

[14] R. Salakhutdinov, A. Mnih, and G. Hinton, "RestrictedBoltzmann MachinesforCollaborative Filtering”, Proc. 24th Annual International Conference on Machine Learning, 2007.

\section{Creative Commons Attribution License 4.0} (Attribution 4.0 International, CC BY 4.0)

This article is published under the terms of the Creative Commons Attribution License 4.0

https://creativecommons.org/licenses/by/4.0/deed.en_US 